\title{
Tile Narrative: Scrollytelling with Grid Maps
}

\author{
Pratap Vardhan* \\ Gramener
}
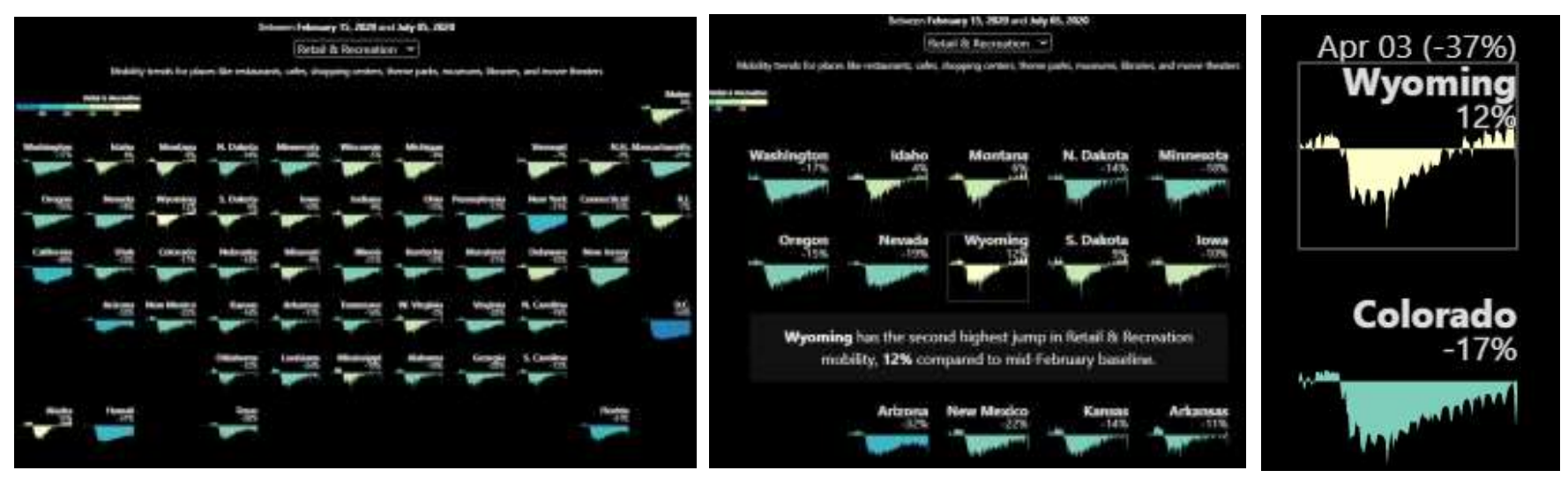

Figure 1: Tile Narrative storytelling format explaining insights from Google's COVID-19 Community Mobility data

\section{Abstract}

This visual case study was built to study the effectiveness of scrollytelling in tandem with tile grid maps. Specifically, we built this interactive based on Google's Community Mobility data measuring people's movement before and during the COVID-19 pandemic. We designed a dynamic and interactive format to display auto-generated narratives synchronized with the context of the grid map. We believe the use of narratives in grid map context will be a useful storytelling format. This paper describes the design decisions that went into building this interactive format.

Index Terms: Human-centered computing-VisualizationVisualization techniques

\section{INTRODUCTION}

While Choropleth maps are the goto method to visualize geographic data, they tend to skew attention towards the bigger states (blocks). Some data stories need equal emphasis for each block (state) and be arranged in way that is close to the actual map representation. Tile grid maps solve this problem. They can be the perfect way avoid the visual imbalances typical to traditional choropleths [1].

\section{Tile Narrative}

Lately, visual scrollytelling [2] has emerged as one of the effective storytelling technique to engage users. Tile grid narrative borrows this format to explain narrative insights from grid maps contextually. In short,

\section{$\underline{\text { Tile Narrative }}=$ Tile grid maps + Narratives + Scrollytelling}

By using these three powerful storytelling techniques, we weave an effective interactive format for geographic data gleaned from

\footnotetext{
*pratap.vardhan@gramener.com
}

google mobility. With the right use of interactions, we engage the reader to spend more time with insights and details that would be shadowed otherwise.

\subsection{Design}

We classified mobility into 5 levels and labelled each one with a different color. The individual chart of each state is assigned a single color based only on the latest data point since multiple colors would have been distracting and latest data seemed more relevant for current situation. As the user scrolls from one insight to the next, the visualization zooms smoothly from the relevant state to the next. We wanted users to absorb narrative insights along with the zoomed version of the states. A curious user can use available tooltips for exploring further details.

\subsection{Interactive Setup}

The tile narrative is setup in following steps - 1) Draw a small multiple tile grid map. 2) Construct narratives. 3) Link each narrative insight to a tile. 4) Trigger an event (state of interest) you scroll to an insight. 5) Zoom the map into the tile of interest. Insights for this dataset are structured in these patterns -- How many regions are better off now compared to the past? What are \#1, \#2 regions with highest gain in mobility? User could also handcraft these insights and decide the flow for the story.

\section{OUtCOME AND FUtURE WORK}

We measured users' interactive behaviour on the page. Most users paused and spent time to read the insights, which likens engagement. Several users indicated they were able to recall the insights for a longer time. An area of improvement would be more controlled and smoother transition through narratives while scrolling the tile grid. We are presently investigating different datasets to understand narrative patterns in order to make this format generically available.

\section{REFERENCES}

[1] D. DeBelius. Let's Tesselate: Hexagons for Tile Grid Maps. http://blog.apps.npr.org/2015/05/11/hex-tile-maps.html 2015.

[2] D. Seyser and M. Zeiller. Scrollytelling - an analysis of visual storytelling in online journalism. In Proc. Int. Conf. Inf. Vis. (IV), 2018 